\title{
Statistical Mechanics of Inverse Halftoning
}

\author{
Yohei Saika \\ Gunma National College of Technology \\ Japan
}

\section{Introduction}

For many years, researchers have investigated information science, such as image analysis (Besag, 1974, Winkler, 1995, Cressie, 1993). Especially, image restoration has been studied as a fundamental problem in information science. In a recent development of this field, theoretical physicists have applied statistical mechanics to information based on analogy between statistical mechanics and Bayesian inference via the maximizer of the posterior (MPM) estimate (Nishimori, 2001). In this field, many techniques in statistical mechanics have been applied to various problems. Following the strategy, the present author has applied statistical mechanics to image restoration using the plane rotator model (Saika \& Nishimori, 2002) and phase retrieval (Saika \& Nishimori, 2005). Recently, statistical mechanical approach for information becomes an established field called as statistical mechanical informatics. Now statistical mechanics has been applied to many problems in various areas, such as information communication and quantum computation.

In print technology, many techniques have been proposed to print images with high quality. Especially, a technique called as digital halftoning (Ulichney, 1987) is essential to convert an original image into a halftone image expressed as a set of black and white dots which are visually similar to the original image through human vision system. A lot of techniques have been proposed for this problem, such as the dither method (Bayer, 1973). On the other hand, the inverse of digital halftoning is called as inverse halftoning and then the purpose is to reconstruct the original image from the halftone image (Miceli, C. M. \& Parker, K. J., 1992). A lot of techniques have been proposed. From the practical point of view, Wong (Wong, 1995) has proposed statistical smoothing to inverse halftoning for halftone images. Then, Stevenson (Stevenson, 1995) has constructed the MAP estimation for halftone dithered images.

In this article, we demonstrate recent development of our researches both on theoretical and practical aspects of inverse halftoning for halftone images obtained by the dither and error diffusion methods (Ulichney, 1987). As shown in Fig. 1, our strategy for this problem is based on the analogy between statistical mechanics and the Bayesian inference via the maximizer of the posterior (MPM) estimate (Fig. 2) and is then to propose the statistical mechanical techniques for this problem. First, we construct a Bayesian probabilistic formulation for inverse halftoning utilizing statistical mechanics of the Q-Ising model (Saika, et al., 2009, Saika \& Okamoto, 2010). Then, we clarify the statistical performance of the present method using both the Monte Carlo simulation for a set of the snapshots of the Q-Ising model and the analytical estimate via the infinite-range model. 


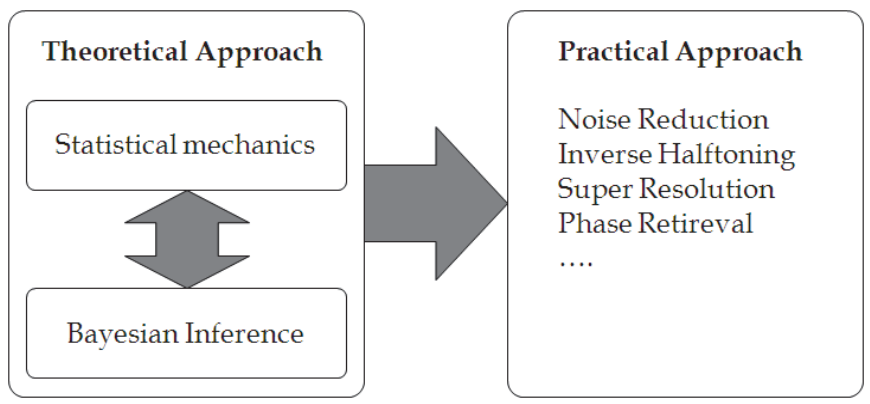

Fig. 1. Statistical mechanical approaches to image processing technology

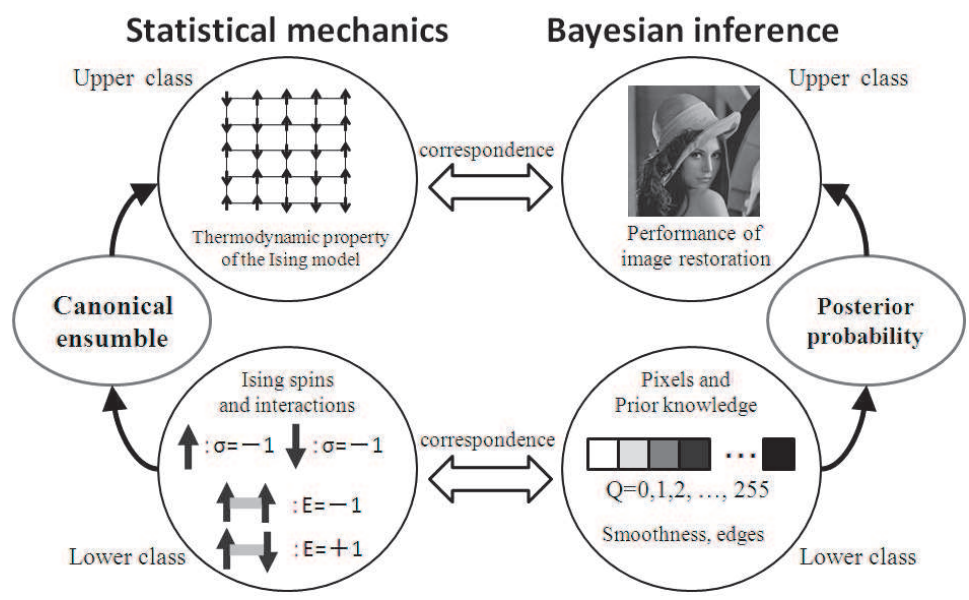

Fig. 2. Analogy between statistical mechanics and Bayesian inference

These estimates clarify that the present method realizes optimal performance around the Bayes-optimal condition. Next, we investigate the practical aspect of this problem by means of the generalized statistical smoothing (GSS) (Saika \& Yamasaki, 2007, Saika, et al., 2010a, 2010b) which is regarded as the generalized MAP estimate corresponding to the deterministic limit of the MPM estimate. Using the numerical simulation for several standard images, we clarify that the GSS is a practically useful method for inverse halftoning, if we set parameters both for edge enhancement and generalized parameter scheduling appropriately. From the above studies, we clarify that statistical mechanical approach and its variants serve various powerful tools for clarifying both theoretical and practical aspects of inverse hafltoning.

\section{Theoretical apsect of inverse halftoning}

In this section, after we show the prescription of statistical mechanics, we then demonstrate the theoretical aspect of our studies (Saika, et al., 2009, Saika \& Okamoto, 2010) for inverse halftoning. Especially, we indicate that the framework of statistical mechanics is available of 
inverse halftoning and that the various techniques in statistical mechanics become powerful tools to clarify the statistical performance of the MPM estimate, such as the Monte Carlo simulation and the analytical estimate via the infinite-range model.

\subsection{Prescription of statistical mechanics}

In this section, we briefly show that statistical mechanics is useful for the clarification of macroscopic properties of many-body systems using knowledge of microscopic elements.

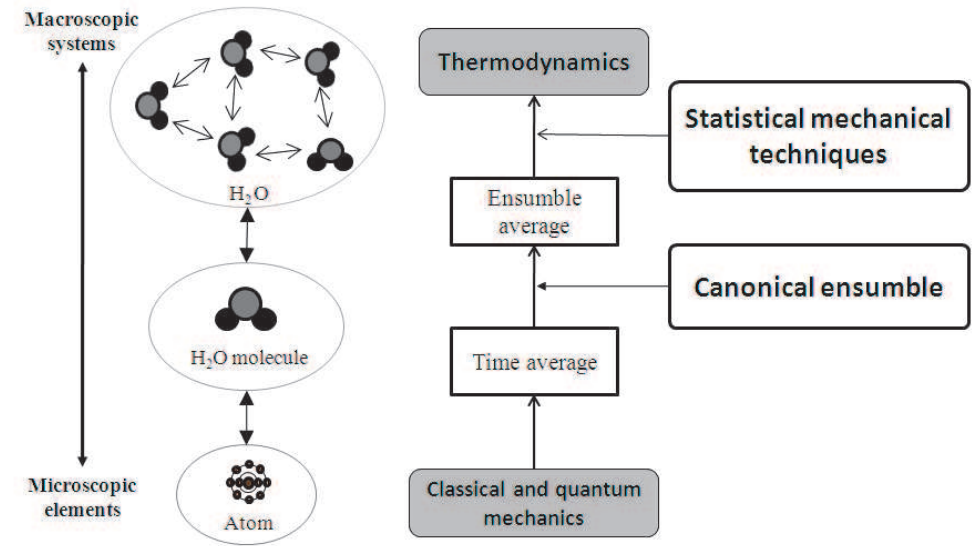

Fig. 3. Prescription of statistical mechanics

As shown in Fig. 3, a goal of statistical mechanics is to clarify the thermodynamic properties of many-body systems starting from the knowledge of interactions between microscopic elements. The general prescription of statistical mechanics is to calculate the thermal average of a physical quantity using the probability distribution

$$
\operatorname{Pr}(\{\xi\})=\frac{1}{Z} \exp \left[-\beta H\left(\left\{S_{i}\right\}\right)\right]
$$

for a given Hamiltonian. Here $\left\{S_{i}\right\}$ represents a set of spin states which are regarded as a typical example of the microscopic elements. Here we take the unit of temperature such that Boltzmann's constant $k_{\mathrm{B}}$ is unity. Then, $\beta$ is the inverse temperature $\beta=1 / T$. The normalization factor $Z$ is called as the partition function:

$$
Z=\sum_{S_{1}= \pm 1} \sum_{S_{2}= \pm 1} \cdots \sum_{S_{N}= \pm 1} e^{-\beta H(\{S\})}
$$

Equation (1) is called the Gibbs-Boltzmann distribution and then $e^{-\beta H}$ is termed the Boltzmann factor. Then, by making use of the Gibbs-Boltzmann distribution, we can estimate the macroscopic quantities, such as the free energy, as

$$
\langle A\rangle=\frac{1}{Z} \sum_{S_{1}= \pm 1} \sum_{S_{2}= \pm 1} \cdots \sum_{S_{N}= \pm 1} A(\{S\}) e^{-\beta H(\{S\})}
$$


utilizing various thermodynamical relations. For instance, the internal energy of the system is obtained by the relation:

$$
\langle E\rangle=\frac{1}{Z} \sum_{S_{1}= \pm 1} \sum_{S_{2}= \pm 1} \cdots \sum_{S_{N}= \pm 1} E(\{S\}) e^{-\beta H(\{S\})}=-\frac{\partial}{\partial \beta} \log Z
$$

using the partition function.

Then, we briefly show the strategy of statistical mechanics to information science and technology. The basic concept of the statistical mechanics to information is based on the analogy between statistical mechanics and the Bayesian inference via the MPM estimate. Following this strategy, the statistical mechanical formulations have been constructed for various problems in information science and technology, such as image restoration and error-correcting codes. Then, researcheres utilize various statistical mechanical techniques, such as the mean-field theory and its variants including the Bethe approximation. Further, we can use these statistical mechanical techniques to clarify the statistical performance, such as the Monte Carlo simulation and the analytical estimate via the infinite-range model.

\subsection{Statistical mechanical formulation for inverse halftoning}

In this section, as shown in Fig. 4, we show the statistical mechanical formulation for inverse halftoning using the Bayesian inference via the MPM estimate for a set of snapshots of the Q-Ising model.

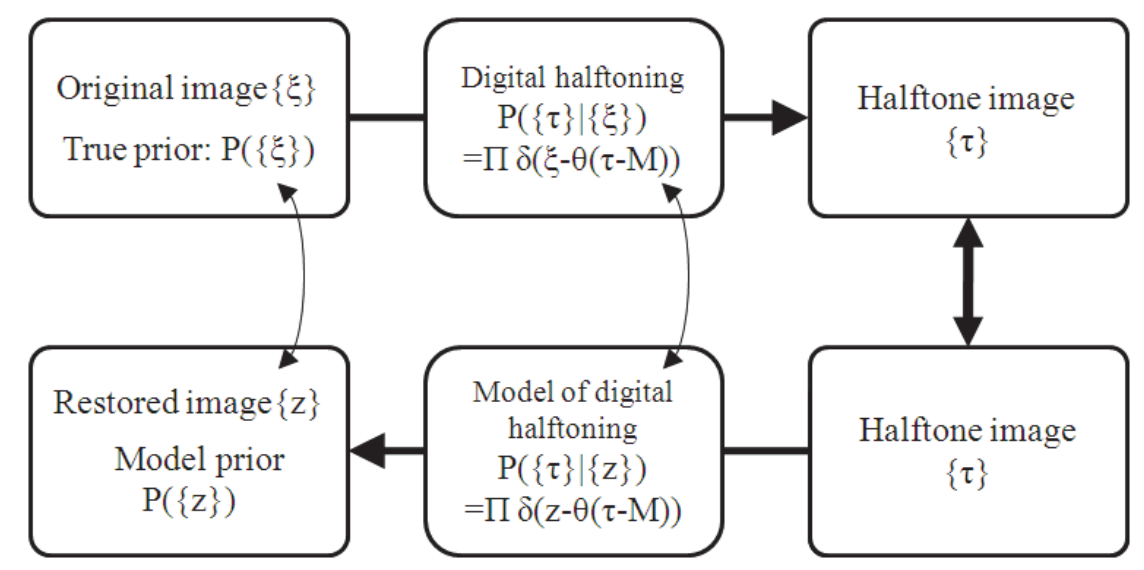

Fig. 4. The reconstruction-based inverse halftoning based on the Bayesian inference.

In this formulation, we first consider the set of original grayscale images $\left\{\xi_{x, y}\right\}\left(\xi_{x, y}=0, \ldots, 255\right.$ and $x, y=1, \ldots, L)$ generated by the assumed true prior which is expressed as the probability distribution:

$$
\operatorname{Pr}(\{\xi\})=\frac{1}{Z_{s}} \exp \left[-\frac{J_{s}}{T_{s}} \sum_{x=1}^{L} \sum_{y=1}^{L}\left[\left(\xi_{x, y}-\xi_{x+1, y}\right)^{2}+\left(\xi_{x, y}-\xi_{x, y+1}\right)^{2}\right]\right]
$$




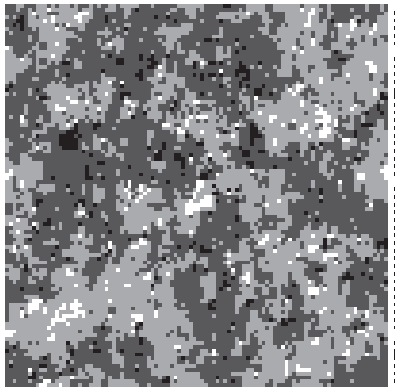

(a)

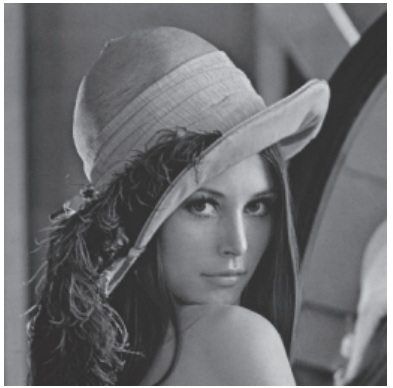

(d)

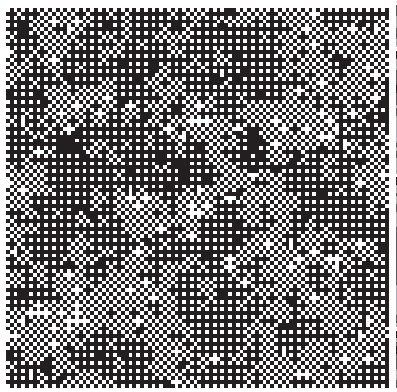

(b)

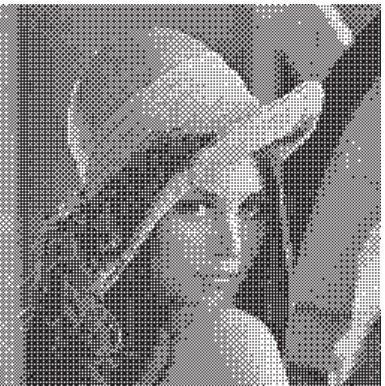

(e)

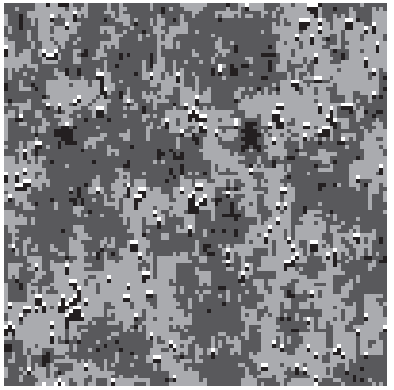

(c)

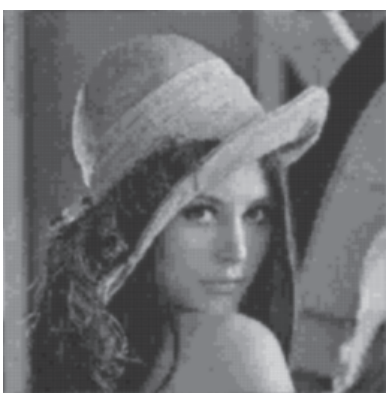

(f)

Fig. 5. (a) a snapshot of the 4-level Q-Ising model with $100 \times 100$ pixels, (b) a halftone image of (a) converted by the dither method via the $2 \times 2$ Bayer-type threshold array, (c) a grayscale image reconstructed from (b) by the MPM estimate under the Bayes-optimal condition, (d) the 256-level standard image "Lena" with $256 \times 256$ pixels, (e) a halftone version of (d) converted by the dither method via the $4 \times 4$ Bayer-type threshold array, (f) a grayscale image reconstructed from (e) by the MPM estimate when $J=5.0$.

Here $J_{s}$ and $T_{s}$ are parameters to generate grayscale images with smooth structures appearing in natural images. A typical pattern of the original images is shown in Fig. 5(a). On the other hand, when we estimate the performance for realistic images we use the 256level standard image "Lena" with $256 \times 256$ pixels in Fig. 5(d).

Then, in the procedure of digital halftoning, we rewrite each original image $\left\{\mathcal{\xi}_{x, y}\right\}$ into a halftone image $\left\{\tau_{x, y}\right\}$ by using the dither method via the $p \times p$ Bayer-type threshold array $\left\{M_{p}\right\}$ in Fig. 5. Here $\tau_{x, y}=0,1$ and $x, y=1, \ldots$, L. The typical threshold arrays are shown in Figs. 6(a) and (b). These threshold arrays $\left\{M_{p}\right\}$ are generated by using the recurrence relation:

$$
\left\{M_{p}\right\}=\left[\begin{array}{cc}
4 M_{p / 2} & 4 M_{p / 2}+2 U_{p / 2} \\
4 M_{p / 2}+3 U_{p / 2} & 4 M_{p / 2}+U_{p / 2}
\end{array}\right]
$$




$$
\left\{M_{2}\right\}=\left[\begin{array}{ll}
0 & 2 \\
3 & 1
\end{array}\right]
$$

Here $U_{n}$ is a $n \times n$ matrix whose all elements are unity. Then, the element of the threshold $M_{p}$ is an integer from 0 to $p^{2}-1$. When we rewrite the original image $\left\{\mathcal{\zeta}_{x, y}\right\}$ into the halftone image $\left\{\tau_{x, y}\right\}$, we first make a one-to-one correspondence between each pixel of the original image $\left\{\mathcal{\xi}_{x, y}\right\}$ and the threshold of the Bayer-type threshold array $\left\{M_{p}\right\}$ using the correspondence relation:

$$
\xi_{x, y} \leftrightarrow M_{x \% p, y \% p}
$$

Here $a \% b$ denotes a surplus which divides $a$ by $b$. Then, we carry out thresholding at each pixel of the original image $\left\{\mathcal{\zeta}_{x, y}\right\}$ by the corresponding threshold $\left\{M_{p}\right\}$ as

$$
\tau_{x, y}=\theta\left(\xi_{x, y}-M_{x \% p, y \% p} \cdot Q / p^{2}-1 / 2\right)
$$

Here $\theta(\ldots)$ is the unit-step function which is defined by

$$
\theta(x)=\left\{\begin{array}{ll}
0 & (x<0) \\
1 & (x>0)
\end{array}\right. \text {. }
$$

The halftone images of the original images in Figs. 5(a) and (d) are shown in Figs. 5(b) and (e). These halftone images are visually similar to the original image if we observe them through the human vision system, although the information on the original images is lost through the halftone procedure.

In the procedure of inverse halftoning, we reconstruct the original image so as to maximize the posterior marginal probability. The pixel value at the $(x, y)$-th pixel of the reconstructed image is given as

$$
\hat{z}_{x, y}=\arg \max _{z_{x, y}} \sum_{\{z\} \neq z_{x, y}} \operatorname{Pr}(\{z\} \mid\{\tau\}) .
$$

The posterior probability in (11) can be estimated based on the Bayes formula:

\begin{tabular}{|l|}
\begin{tabular}{|c|c|c|c|c|c|c|c|c|c|}
\hline 0 & 32 & 8 & 40 & 2 & 34 & 10 & 42 \\
\hline 48 & 16 & 56 & 24 & 50 & 18 & 58 & 26 \\
\hline 12 & 44 & 4 & 36 & 14 & 46 & 6 & 38 \\
\hline 60 & 28 & 52 & 20 & 62 & 30 & 54 & 22 \\
\hline 0 & 8 & 2 & 10 \\
\hline 12 & 4 & 14 & 6 \\
\hline 3 & 11 & 1 & 9 \\
\hline 15 & 7 & 13 & 5 \\
\hline 3 & 35 & 11 & 43 & 1 & 33 & 9 & 41 \\
\hline 51 & 19 & 59 & 27 & 49 & 17 & 57 & 25 \\
\hline 15 & 47 & 7 & 39 & 13 & 45 & 5 & 37 \\
\hline 63 & 31 & 55 & 23 & 61 & 29 & 53 & 21 \\
\hline
\end{tabular}
\end{tabular}

(a)

Fig. 6. (a) the $4 \times 4$ Bayer-type threshold array, (b) the $8 \times 8$ Bayer-type threshold array 


$$
\operatorname{Pr}(\{z\} \mid\{\tau\})=\frac{\operatorname{Pr}(\{z\}) \operatorname{Pr}(\{\tau\} \mid\{z\})}{\sum_{\{\tau\}} \operatorname{Pr}(\{z\}) \operatorname{Pr}(\{\tau\} \mid\{z\})}
$$

using the assumed model prior and the likelihood. In this study, we assume the model prior which is expressed as the probability distribution:

$$
\operatorname{Pr}(\{z\}) \propto \exp \left[-\frac{J}{T_{m}} \sum_{x=1}^{L} \sum_{y=1}^{L}\left[\left(z_{x, y}-z_{x+1, y}\right)^{2}+\left(z_{x, y}-z_{x, y-1}\right)^{2}\right]\right]
$$

so as to enhance smooth structures in the patterns of the reconstructed image. Then, in order to construct the Bayes-optimal solution, we consider the model prior which has the same form as the assumed true prior in (5). Then, we use the likelihood which is expressed as the conditional probability representing the dither method via the Bayer-type threshold array as

$$
\operatorname{Pr}(\{z\} \mid\{\tau\})=\prod_{x=1}^{L} \prod_{y=1}^{L} \delta\left(\tau_{x, y}, \theta\left(z_{x, y}-M_{x \% p, y \% p} \cdot Q / p^{2}-1 / 2\right)\right)
$$

In this study, the reconstructed image is obtained by

$$
\hat{z}_{x, y}=\Theta\left(\bar{z}_{x, y}\right),
$$

where

$$
\begin{gathered}
\bar{z}_{x, y}=\sum_{\{z\}} z_{x, y} \operatorname{Pr}\left(\{z\} \mid\left\{\tau^{p^{2}}\right\}\right), \\
\Theta(x)=\sum_{k=0}^{Q} \theta\left(x-k+\frac{1}{2}\right)-\theta\left(x-k-\frac{1}{2}\right) .
\end{gathered}
$$

When we estimate the performance of the present method for the realistic image, we evaluate the mean square error (MSE) defined by

$$
M S E=\frac{1}{L^{2}} \sum_{x=1}^{L} \sum_{y=1}^{L}\left(\hat{z}_{x, y}-\xi_{x, y}\right)^{2} .
$$

Then, when we estimate the statistical performance, we evaluate the MSE averaged over the set of the original images $\left\{\xi_{x, y}\right\}$ as

$$
M S E=\sum_{\{\xi\}} \operatorname{Pr}(\{\xi\}) \frac{1}{L^{2}} \sum_{x=1}^{L} \sum_{y=1}^{L}\left(\hat{z}_{x, y}-\xi_{x, y}\right)^{2} .
$$

\subsection{Statistical performance}

In this section, we indicate that the Monte Carlo simulation is useful for clarifying the statitstical performance of the MPM estimate. When we investigate the statistical performance for the set of the snapshots of the Q-Ising model. As shown in Fig. 5(a), we numerically estimate the statistical performance for the set of the snapshots of the Q-Ising 
model. These images are generated by the assumed true prior expressed by the Boltzmann factor of the Q-Ising model when we set to $\mathrm{Q}=4$ and $J_{\mathrm{s}}=T_{\mathrm{s}}=1$. Then, each original image is converted into the halftone image by the dither method via the $2 \times 2$ Bayer-type threshold array. Then, when we carry out the Monte Carlo simulation, we use the Metropolis algorithm with 20000 Monte Carlo steps.

In order to clarify the statistical performance for the set of the Q-Ising model, we numerically estimate how the MSE depends on the parameter $T_{m}$ when $J=1$. As shown in Fig. 7, the Monte Carlo simulations clarify that optimal performance is realized around the Bayes-optimal condition, $T_{m}=T_{\text {s. }}(=1)$ within statistical uncertainty. This result also means that the optimal performance of the MPM estimate is as well as that of the MAP estimate, if we set the parameters appropriately. Here, we denote the MAP estimate as the $T_{m} \rightarrow 0$ limit of the MPM estimate.

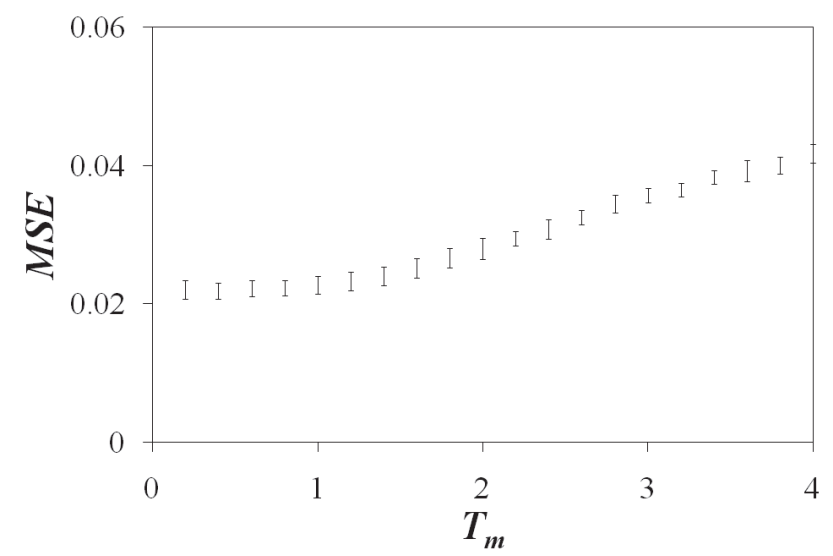

Fig. 7. The MSE as a function of $T_{m}$ obtained by the Monte Carlo simulation for the set of the snapshots of the Q-Ising model.

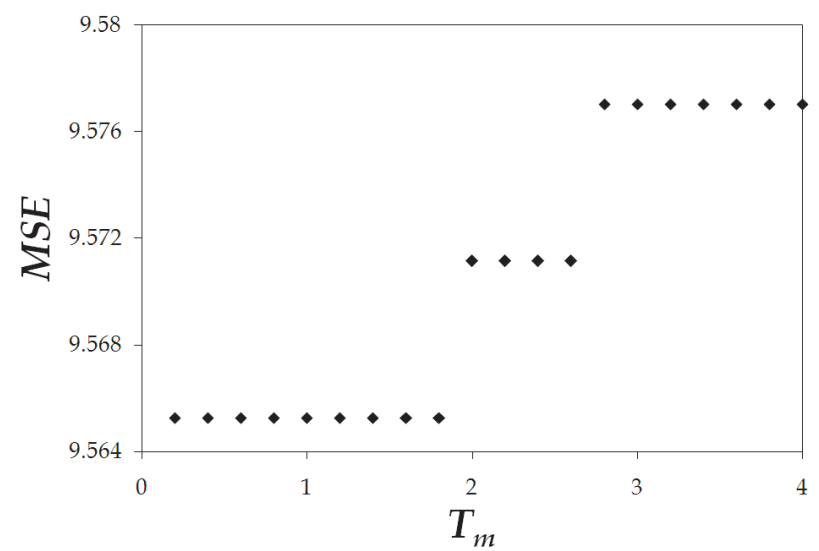

Fig. 8. The MSE as a function of the parameter $T_{m}$ obtained by the analytical estimate using the infinite-range model. 


\subsection{Analytical estimate via the infinite-range model}

In this section, we clarify that the analytical estimate via the infinite-range model is useful for the estimation of the statistical performance of the MPM estimate averaged over the set of the snapshots of the Q-Ising model. For convenience, we here use the language in the field of statistical mechanics.

In order to estimate the statistical performance, we first introduce the infinite-range versions of the model and true priors:

$$
\begin{array}{r}
\operatorname{Pr}\left(\left\{\xi_{i}\right\}\right)=\frac{1}{Z_{s}} \exp \left[-\frac{J_{s}}{T_{s}} \sum_{i<j}\left(\xi_{i}-\xi_{j}\right)^{2}\right], \\
\operatorname{Pr}\left(\left\{z_{i}\right\}\right)=\frac{1}{Z_{m}} \exp \left[-\frac{J_{m}}{T_{m}} \sum_{i<j}\left(z_{i}-z_{j}\right)^{2}\right],
\end{array}
$$

both of which are assumed to approximate the assumed model and true priors in two dimensions. Then, based on the saddle-point conditions on the free energy (Nishimori, 2001, Saika, et al., 2009), we can derive the self-consistent equations on $m_{0}$ and $m$ as

$$
\begin{gathered}
m_{0}=\frac{1}{Z_{s}} \sum_{\xi=0}^{Q-1} \xi \exp \left[\beta_{s}\left(2 m_{0} \xi-m_{0}^{2}\right)\right] \\
m=\frac{1}{Z_{s}} \sum_{\xi=0}^{Q-1} \exp \left[\beta_{s}\left(2 m_{0} \xi-m_{0}^{2}\right)\right]\left[\frac{\sum_{z=0}^{Q-1} \prod_{k=1}^{L_{M}^{2}} \delta\left(\xi, \theta\left(z-k \frac{k Q}{L_{M}^{2}}\right) \exp \left[\beta_{m}\left(2 m z-m_{0}^{2}\right)\right] z\right.}{\sum_{z=0}^{Q-1} \prod_{k=1}^{L_{M}^{2}} \delta\left(\xi, \theta\left(z-k \frac{k Q}{L_{M}^{2}}\right) \exp \left[\beta_{m}\left(2 m z-m_{0}^{2}\right)\right]\right.}\right] \\
Z_{s}=\sum_{\xi=0}^{Q-1} \exp \left[\beta_{s}\left(2 m_{0} \xi-m_{0}^{2}\right)\right]
\end{gathered}
$$

using the infinite-range versions of the model and true priors. In above equations, $\beta_{\mathrm{s}}=1 / T_{\mathrm{s}}$ and $\beta_{m}=1 / T_{m}$ respectively. By making use of the solutions $m_{0}$ and $m$ on the self-consistent equations in (22)-(24), we can estimate the MSE:

$$
M S E=\frac{\sum_{\xi=0}^{Q-1} \exp \left[\beta_{s}\left(2 m_{0} \xi-m_{0}^{2}\right)\right]}{\sum_{\xi=0}^{Q-1} \exp \left[\beta_{s}\left(2 m_{0} \xi-m_{0}^{2}\right)\right]}(\Theta(\hat{z})-\xi)^{2},
$$

where

$$
\hat{z}=\frac{\sum_{z=0}^{Q-1} \prod_{k=1}^{L_{M}^{2}} \delta\left(\xi, \theta\left(z-k \frac{k Q}{L_{M}^{2}}\right) \exp \left[\beta_{m}\left(2 m z-m_{0}^{2}\right)\right] z\right.}{\sum_{z=0}^{Q-1} \prod_{k=1}^{L_{M}^{2}} \delta\left(\xi, \theta\left(z-k \frac{k Q}{L_{M}^{2}}\right) \exp \left[\beta_{m}\left(2 m z-m_{0}^{2}\right)\right]\right.}
$$


which is averaged over the true prior.

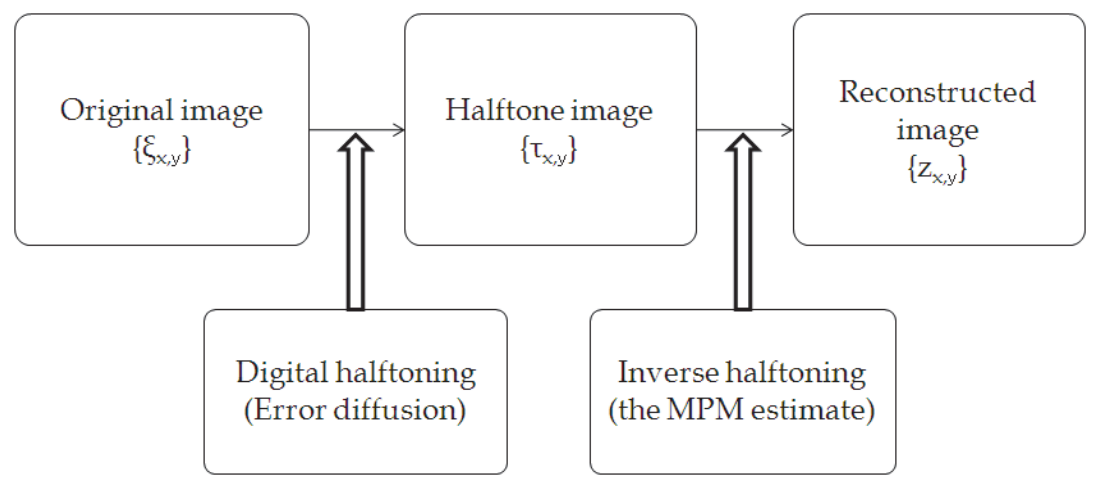

Fig. 9. The general formulation of inverse halftoning for the halftone image converted by the error diffusion method

As shown in Fig. 8, the analytical estimate via the infinite-range model clarifies that the MPM estimate achieves the optimal performance around the Bayes-optimal condition $T_{m}=T_{s}$ $(=1)$ without the statistical uncertainty. This shows that the results of the Monte Carlo simulation are qualitatively confirmed by the analytical estimate via the infinite-range model.

\subsection{Realistic image}

In this section, we indicate that the present method is also available of inverse halftoning for realistic images. Especially, we numerically estimate the performance of the MPM estimate using the Monte Carlo simulation for the 256-level standard image "Lena" with $256 \times 256$ pixels. As shown in Fig. 5 (f), we find that the present method is effective for inverse halftoning, if we assume the parameters appropriately.

\section{Practical apsect of inverse halftoning}

\subsection{Generalized statistical smoothing}

In this section, we indicate that the practically useful technique can be constructed as the generalized MAP estimate corresponding to the deterministic limit of the MPM estimate. In this article, the technique called as the GSS (Saika \& Yamasaki, 2007, Saika et al., 2010a, Saika et al., 2010b) is constructed by introducing the edge enhancement and the generalized parameter scheduling into the MAP estimate. Here we show how to use the GSS to inverse halftoning for the halftone image which is converted by the error diffusion method (Floyd and Steinberg, 1975).

As shown in Fig. 9, we show the general formulation of the GSS to inverse halftoning for the halftone image converted by the error diffusion method. Then, we indicate the performance measure which utilizes the MTF function of the human vision system.

In this formulation, we first consider an original grayscale image $\left\{\xi_{x, y}\right\}\left(\xi_{x, y}=0, \ldots, 255\right.$, $x, y=0, \ldots, \mathrm{L}-1)$ on the square lattice. Here the pixel value $\xi_{x, y}$ represents the brightness at the 
$(x, y)$-th site on the square lattice. In this study, we use several 256-level standard image, such as "Lena" with $256 \times 256$ pixels in Fig. 10(a). Then, in the procedure of digital halftoning, we convert the original image $\left\{\mathcal{\xi}_{x, y}\right\}$ into a halftone image $\left\{\tau_{x, y}\right\}\left(\tau_{x, y}=0, \mathrm{Q}-1\right.$, $x, y=0, \ldots, \mathrm{L}-1)$ by using the error diffusion method. The block diagram of this method is shown in Fig. 11 and the Floyd-Steinberg kernel is shown in Fig. 12. Then, as shown in Fig. 10 (b), the density of the black and white dots of the halftone image approximate the gray levels of the original image and are visually similar to the original image through the human vision system.

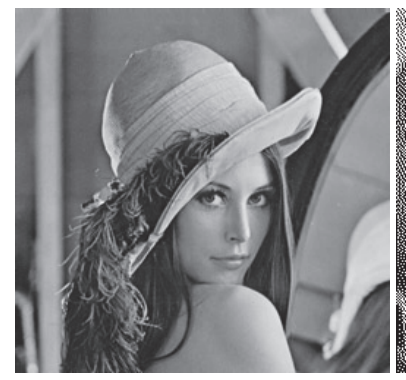

(a)

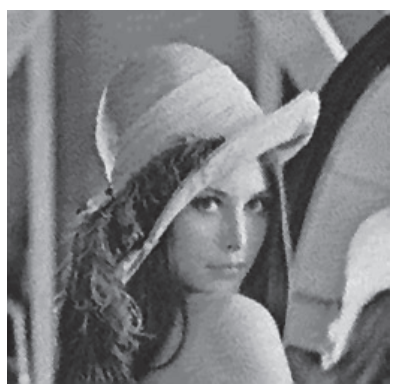

(d)

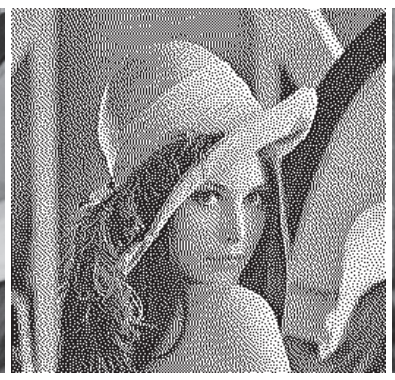

(b)

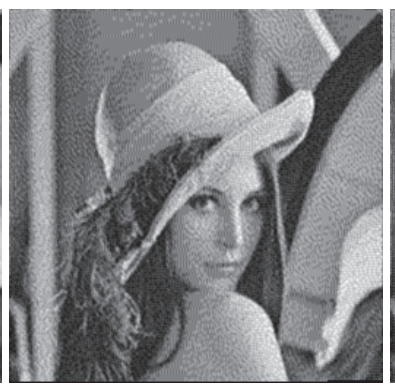

(e)

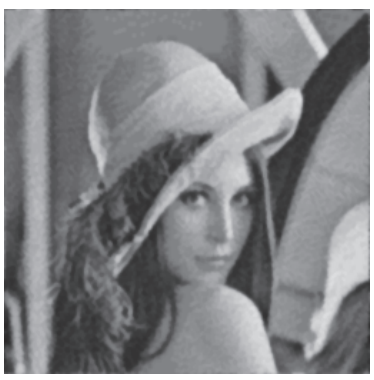

(c)

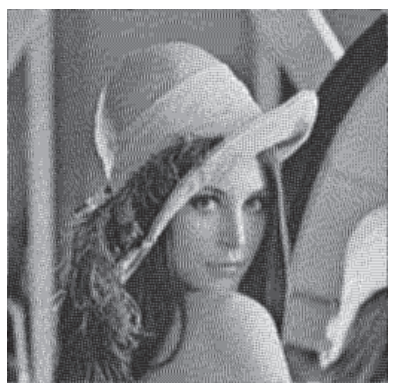

(f)

Fig. 10. (a) the 256-level standard image "Lena" with $256 \times 256$ pixels, (b) the halftone image converted from the standard image (a) by the error diffusion using the Floyd-Steinberg's kernel, (c) the restored image due to the GSS when $\kappa=2.5$ and $D=0$ (d) the restored image using the GSS when $\kappa=2.5$ and $D=25$, (e) the restored image obtained by the Gaussian filter, (f) the restored image obtained by the average filter.

Next, we carry out inverse halftoning by using the GSS constructed by introducing both the edge enhancement and the generalized parameter scheduling into the statistical smoothing originally proposed by Wong (Wong, 1995). Here, we construct this method so as to achieve the optimal performance when we observe images through the MTF function of the human vision system. We carry out the GSS by repeating fundamental processes by 5 times. Then, each fundamental process composed of two parts is carried out through pixel by pixel in a raster scan. At the first part, we calculate a mean: 


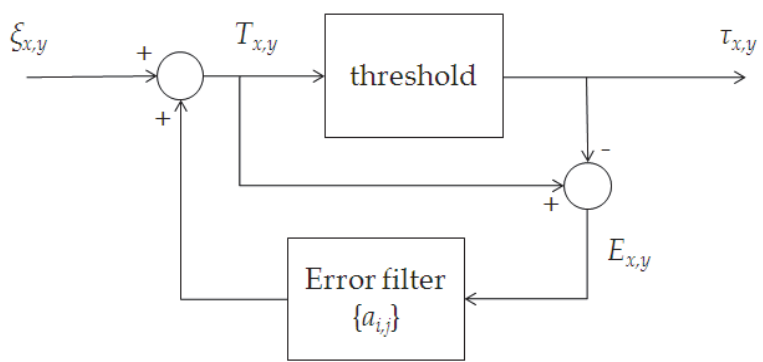

Fig. 11. Block diagram of the error diffusion method, where $\left\{a_{i, j}\right\}$ is the kernel of the error diffusion method

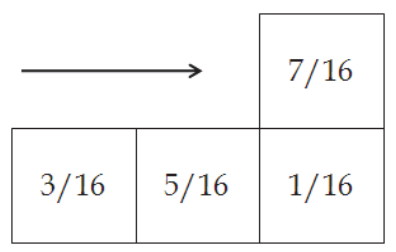

Fig. 12. Floyd-Steinberg's kernel used in the error diffusion method

$$
\mu_{m, n}=\sum_{i, j \in R_{m, n}} a_{i, j} x_{i, j}^{\text {old }}
$$

which is averaged over the pixels in the region $R_{m, n}$ which includes the $(m, n)$-th site and the $\left(m+\delta_{x}, n+\delta_{y}\right)$-sites $\left(\delta_{x}, \delta_{y}=-1,0,1\right)$ which hold the condition:

$$
\left|x_{m+\delta_{x}, n+\delta_{y}}^{\text {old }}-x_{m, n}^{\text {old }}\right|<D \text {. }
$$

Here, $D$ is the threshold to detect edges appearing in original images and should be set respective of the choice of the original image. Then, $\{a i, j\}$ is the kernel of the conventional Gaussian filter. We note that the present method is regarded as the original statistical smoothing, if $D=256$. On the other hand, as clearly seen from eq. (28), smoothing does not work if we set to $D=0$. In this procedure, we then compute a measure $v_{\mathrm{m}, \mathrm{n}}$ given by the standard deviation:

$$
v_{m, n}=\left[\frac{1}{\left\|R_{m, n}\right\|} \sum_{(i, j) \in R_{m, n}}\left|x_{i, j}^{\text {old }}-\mu_{i, j}\right|^{r}\right]^{1 / r} .
$$

which is averaged over the pixels in the region $R_{m, n}$. Here $\left\|R_{m, n}\right\|$ is the number of the pixels in the region $R_{m, n}$. Then, the second step of the core process is the smoothing procedure as

$$
x_{m, n}^{\text {new }}= \begin{cases}\mu_{m, n}+\gamma v_{m, n} & \text { if } x_{m, n}^{\text {old }}>\mu_{m, n}+\gamma v_{m, n} \\ \mu_{m, n}-\gamma v_{m, n} & \text { if } x_{m, n}^{\text {old }}<\mu_{m, n}-\gamma v_{m, n} \\ x_{m, n}^{\text {old }} & \text { otherwise }\end{cases}
$$




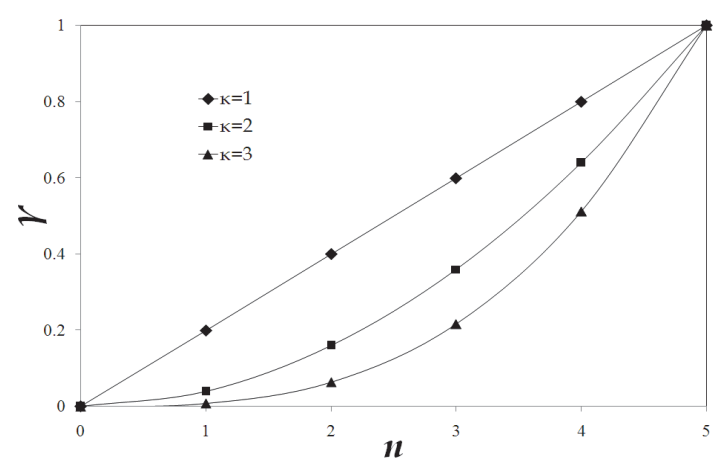

Fig. 13. Generalized parameter scheduling in the GSS.

Here $\gamma$ is a positive parameter which evolves following the schedule:

$$
\gamma=\left(\frac{n}{5}\right)^{\kappa}
$$

where $n$ is the positive integer from 1 to 5 . This schedule is also shown in Fig. 11 . If $\kappa=1$, this method is same as the original statistical smoothing proposed by Wong (Wong, 1995). Then, if $\gamma=0$, the present method is regarded as the conventional smoothing filter which is characterized by the kernel $\left\{a_{i, j}\right\}$.

When we estimate the performance of the GSS for the standard image, as shown in Fig. 14, we use the mean square error between original and reconstructed images both of which are modulated by the MTF function:

$$
H\left(k_{x}, k_{y}\right)=\left\{\begin{array}{cc}
5.05 \exp \left[-1.38 \sqrt{k_{x}^{2}+k_{y}^{2}}\right]\left(1-\exp \left[-0.1 \sqrt{k_{x}^{2}+k_{y}^{2}}\right]\right) \\
\left(5 \leq \sqrt{k_{x}^{2}+k_{y}^{2}}\right) \\
1 & \left(0<\sqrt{k_{x}^{2}+k_{y}^{2}}\right)
\end{array}\right.
$$

which approximates the human vision system. That is, we numerically estimate the performance measure which is expressed as

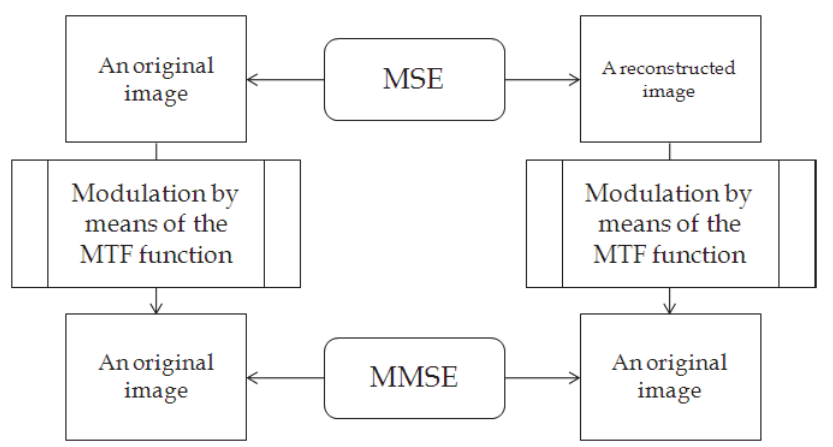

Fig. 14. Performance measure via the MTF function approximating the human vision system 


$$
\text { MMSE }=\frac{1}{L^{2}} \sum_{x=0}^{L} \sum_{y=0}^{L}\left|\hat{z}_{x, y}-\hat{\xi}_{x, y}\right|^{2}
$$

where

$$
\begin{gathered}
\hat{z}_{x, y}=\sum_{x=1}^{L} \sum_{Y=1}^{L} \exp \left[-i\left(k_{x} x+k_{y} y\right)\right] \hat{z}_{k_{x}, k_{y}}, \\
\hat{z}_{k_{x}, k_{y}}=z_{k_{x}, k_{y}} H\left(k_{x}, k_{y}\right), \\
\hat{z}_{k_{x}, k_{y}}=\frac{1}{L^{2}} \sum_{x=1}^{L} \sum_{Y=1}^{L} \exp \left[i\left(k_{x} x+k_{y} y\right)\right] \hat{z}_{k_{x}, k_{y}}
\end{gathered}
$$

For convenience, we note the performance measure as the MMSE in the following part of this paper.

\subsection{Numerical simulation}

In this section, using the numerical simulation for the 256-level standard image "Lena" with $256 \times 256$ pixels (Fig. 10(a)), we estimate the performance of the GSS to inverse halftoning for the halftone images converted by the error diffusion method via the Floyd-Steinberg's kernel. When we estimate the performance of the GSS for inverse halftoning, we numerically estimate the MMSE between original and reconstructed images.

First, in order to clarify the efficiency of the edge enhancement and the generalized parameter scheduling, we evaluate how the MMSE depends on the threshold $D$ for the edge enhancement and the parameter $\kappa$ for the generalized parameter scheduling. Using the numerical simulation for the 256-level halftone image "Lena" with $256 \times 256$ pixels, as shown in Fig. 15, we find that the GSS achieves the optimal performance, if we set to $D=25$ and $\kappa=2.5$ for the 256-level standard image "Lena" with $256 \times 256$ pixels. Then, as shown in Figs. 10 (d) and (f), . We also find that the generalized parameter scheduling due to the parameter $\kappa$ appropriately, and that the GSS reconstructs original image with higher image quality than the conventional average and Gaussian filters.

These results indicate that the performance of the statistical smoothing is improved by introducing appropriate models of the edge enhancement and the generalized parameter scheduling and that the practically useful technique via the GSS can be constructed as the extension of the statistical mechanical method which corresponds to the Bayesian inference via the MPM estimate.

\section{Conclusion}

In above sections, we have shown our researches on both theoretical and practical aspects of inverse halftoning using the statistical mechanical method and the practical filter via the GSS. First, on the basis of the statistical mechanics of the Q-Ising model, we have investigated the theoretical aspect on inverse halftoning utilizing the Bayesian inference via the MPM estimate. Then, we have investigated the statistical performance using the Monte Carlo simulation for the set of the snapshots of the Q-Ising model. The simulations have found that the optimal performance is realized around the Bayes-optimal condition within statistical 


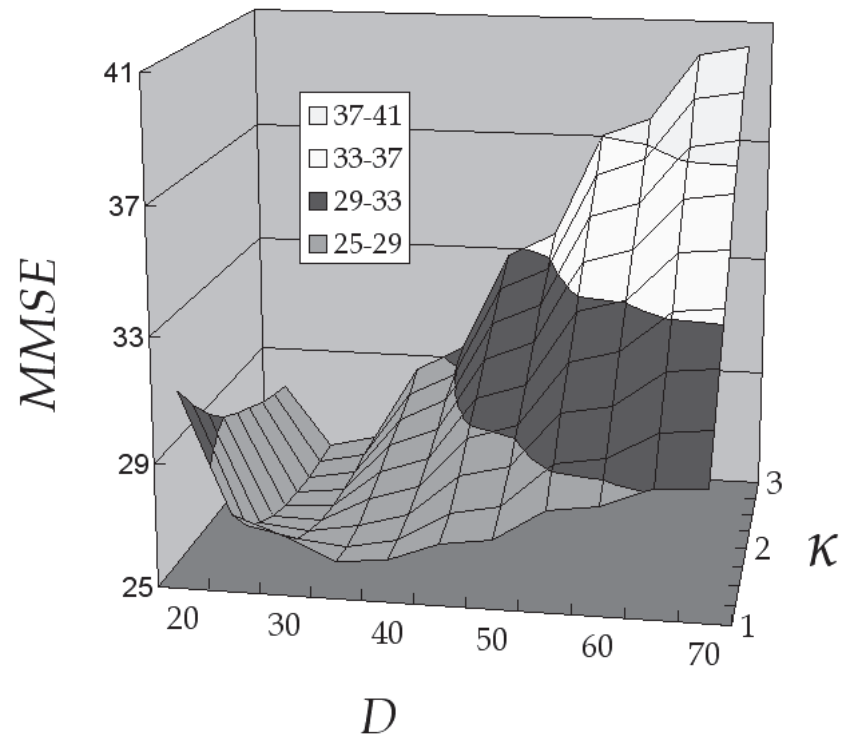

Fig. 15. MMSE as a function of the parameter $\mathrm{D}$ and $\mathrm{\kappa}$ for the 256-level standard image "Lena" with $256 \times 256$ pixels

uncertainty and that it is almost same as the performance of the MAP estimate corresponding to the deterministic limit of the MPM estimate. These results are qualitatively confirmed by the analytical estimate via the infinite-range model. Also we have clarified that the MPM estimate reconstructs original images accurately, if we assume the appropriate model of the true prior. These results have clarified that prior information on original images are important to achieve inverse halftoning with high image quality. Next, we have investigated the practical aspect of inverse halftoning for the halftone image converted by the error diffusion method. In this study, we have constructed the GSS which is regarded as the generalized MAP estimate corresponding to the deterministic limit of the MPM estimate. Here, in order to realize the high performance technique for inverse halftoning, we have proposed the GSS which is constructed by introducing both the edge enhancement and the generalized parameter scheduling into the conventional MAP estimate. Using the numerical simulation for the 256-level standard image, we have clarified that the high performance is achieved by tuning both the threshold for the edge enhancement and the parameter for the generalized parameter scheduling. The above studies have indicated that the theoretical study based on the statistical mechanics gives useful suggestions to construct the practically useful technique based on the MAP estimate.

In the previous researches, we have clarified that the statistical mechanics serves various powerful tools to investigate the problem of inverse halftoning by making use of various techniques, such as the Monte Carlo simulation and the analytical estimate via the infinite-range model, and that the statistical mechanics serves practical and useful techniques based on the MAP estimate. As a future problem, we are going to construct the statistical mechanical techniques based on the Bayesian inference via the MPM estimate 
by utilizing the knowledge obtained from the practical approach via the GSS for inverse halftoning.

\section{References}

Bayer, B. E. (1973). An optimum method for two-level rendition continuous tone pictures, ICC CONF. RECORD, pp. 11-15, 1973

Besag, J. (1974). Spatal Interaction and Statistical Analysis of Lattice System, the Journal of the. Royal. Statistics. Society, Series B, 36(2), pp. 192-236, 1974

Cressie, N. A. C. (1993). Statistics for Spatial Data, Wiley, New York, 1993

Floyd, R. W. \& Steinberg, L. (1975). Adaptive algorithm for spatioal gray scale, Proceedings of the SID International Symposium Digest of Technical Papers, pp. 36-37, 1975

Miceli, C. M. \& Parker, K. J. (1992). Inverse Halftoning, J. Electron Imaging, Vol. 1, pp. 143151,1992

Nishimori, H. (2001). Statistical Physics of Spin Glasses and Information Processing; An Introduction, Oxford, London, 2001

Saika, Y. \& Nishimori, H. (2002). Statistical Mechanics of Image Restoration by the Plane Rotator Model, the Journal of the Physical Society of Japan, Vol. 71, pp. 1052-1058, 2002

Saika, Y. \& Nishimori, H. (2005). Statistical mechanical Approaches to the Problem of Phase Retrieval by the Q-Ising Model, Progress of Theoretical Physics Suppliment, Vol. 157, pp.292-295, 2005

Saika, Y. \& Yamasaki, T. (2007). Generalized Statistical Smoothing to the Problem of Inverse Halftoning for Error Diffusion, Proceedings of the ICCAS 2007, pp. 781-784, 2007

Saika, Y.; Inoue, J.; Tanaka, H. \& Okada, M. (2009). Bayes-optimal solution to inverse halftoning based on statistical mechanics of the Q-Ising model, Central European Journal of Physics, Vol. 7(3), pp. 444-456

Saika, Y. \& Okamoto, K. (2010). Probabilistic Modeling to Inverse Halftoning using Edge Preserving Prior, Proceedings of the SPPRA 2010, pp. 318-321, 2010

Saika, Y. Sugimoto, K. \& Okamoto, K. (2010). Performance Estimation of Generalized Statistical Smoothing to Inverse Halftoning based on the MTF Function of Human Eyes, Proceedings of the ICA3PP 2010, pp. 358-367, 2010

Saika, Y., Okamoto, K. \& Nakagawa, M. (2010). Generalized Parameter Scheduling and Edge Enhancement in Statistical Smoothing Inverse Halftone Filter, Proceedings of the CIVS 2010, pp. 2010, pp. 142-145, 2010

Stevenson, R. (1995). Inverse Halftoning via MAP Estimation, IEEE Trans. Image Processing, Vol. 6, pp. 574-583, 1995

Ulchney, R. (1987). Digital Halftoning, The MIT Press, Cambridge, Massachusetts, London, England 1987

Winkler, G. (1995). Image Analysis, Random fields and Dynamic Monte Carlo Methods, A Mathematical Introduction, Springer-Verlag, Berlin, 1995

Wong, P. W. (1995). Inverse Halftoning and Kernel Estimation for Error Diffusion, IEEE Trans. Image Processing, 4, pp. 486-498, 1995 


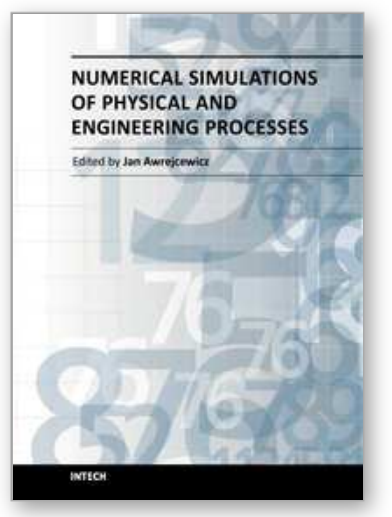

\author{
Numerical Simulations of Physical and Engineering Processes \\ Edited by Prof. Jan Awrejcewicz
}

ISBN 978-953-307-620-1

Hard cover, 594 pages

Publisher InTech

Published online 26, September, 2011

Published in print edition September, 2011

Numerical Simulations of Physical and Engineering Process is an edited book divided into two parts. Part I devoted to Physical Processes contains 14 chapters, whereas Part II titled Engineering Processes has 13 contributions. The book handles the recent research devoted to numerical simulations of physical and engineering systems. It can be treated as a bridge linking various numerical approaches of two closely interrelated branches of science, i.e. physics and engineering. Since the numerical simulations play a key role in both theoretical and application oriented research, professional reference books are highly needed by pure research scientists, applied mathematicians, engineers as well post-graduate students. In other words, it is expected that the book will serve as an effective tool in training the mentioned groups of researchers and beyond.

\title{
How to reference
}

In order to correctly reference this scholarly work, feel free to copy and paste the following:

Yohei Saika (2011). Statistical Mechanics of Inverse Halftoning, Numerical Simulations of Physical and Engineering Processes, Prof. Jan Awrejcewicz (Ed.), ISBN: 978-953-307-620-1, InTech, Available from: http://www.intechopen.com/books/numerical-simulations-of-physical-and-engineering-processes/statisticalmechanics-of-inverse-halftoning

\section{INTECH}

open science | open minds

\author{
InTech Europe \\ University Campus STeP Ri \\ Slavka Krautzeka 83/A \\ 51000 Rijeka, Croatia \\ Phone: +385 (51) 770447 \\ Fax: +385 (51) 686166 \\ www.intechopen.com
}

\author{
InTech China \\ Unit 405, Office Block, Hotel Equatorial Shanghai \\ No.65, Yan An Road (West), Shanghai, 200040, China \\ 中国上海市延安西路65号上海国际贵都大饭店办公楼 405 单元 \\ Phone: +86-21-62489820 \\ Fax: +86-21-62489821
}


(C) 2011 The Author(s). Licensee IntechOpen. This chapter is distributed under the terms of the Creative Commons Attribution-NonCommercialShareAlike-3.0 License, which permits use, distribution and reproduction for non-commercial purposes, provided the original is properly cited and derivative works building on this content are distributed under the same license. 